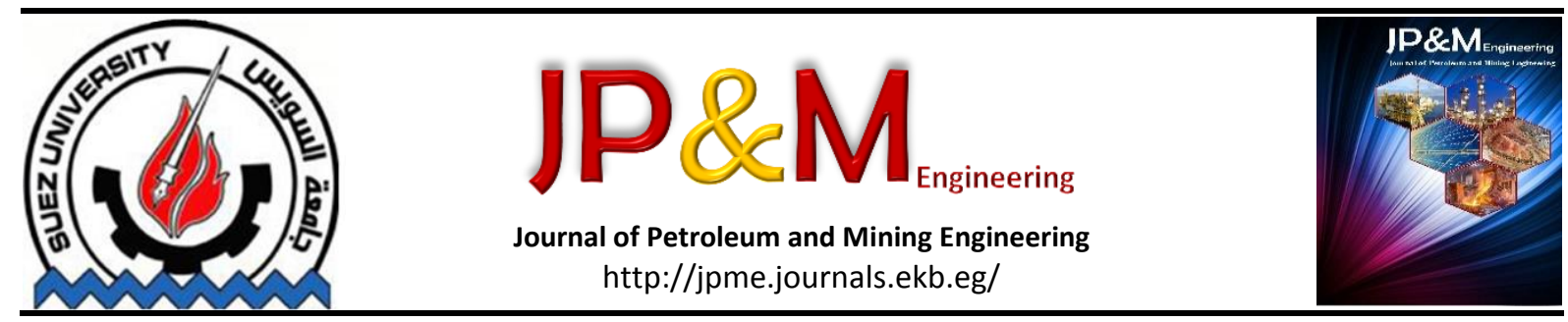

\title{
Modification of Relative Permeability Curves by ultrasonic Applications
}

\author{
Adel M. Salem ${ }^{a *}$, Mohamed F. Snosy ${ }^{b}$, Ali Abbas ${ }^{a}$, and Ahmed Salah ${ }^{c}$ \\ ${ }^{a}$ Faculty of Petroleum and Mining Engineering, Suez University (SU), Egypt

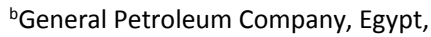 \\ cPetrobel Company, Egypt \\ *Corresponding author e-mail: adel.salem@suezuni.edu.eg
}

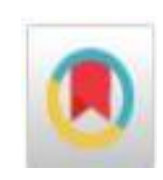

\begin{abstract}
Ultrasonic waves have been used for improved oil recovery especially from the marginal well in so many areas all over the world. The main mechanism is to supply oil molecules by energy to overcome capillary forces there by restructuring the relative permeability curves and increasing the oil mobility. Monitoring these changes is very important for increasing the oil mobility even after residual oil saturation achieved. The present work investigates the laboratory effects of ultrasonic waves on the relative permeability curves. Five sandstone and carbonate cores were used from Egyptian reservoirs. Their permeabilities range from 67 to 460 md. Acoustic ultrasonic waves of $500 \mathrm{KHZ}$ have been applied. At this frequency, the fluid vibrates out of phase with the solid and is forced out through the pore structure in the agglomerate. This relative fluid motion exerts high viscous stresses at the particle-particle contact points which leads to fracture of the agglomerate and the dispersion of the individual particles. This interaction causes changes in relative permeability of the rock to oil and water. Therefore, the results showed that applying ultrasonic waves has a higher effect in permeability reservoirs (76 md to $460 \mathrm{md}$ ) and can mobilize additional quantity of crude oil. The fractional flow curve changes are also addressed and analyzed after wave applications. The aim of this research is to investigate the effect of the ultrasonic wave as a new proposed method to improve oil recovery by changing the relative permeability curves of the reservoirs.
\end{abstract}

Ultrasonic Waves; EOR/IOR; Relative Permeability Curve; Recovery Factor; Core Sample

\section{Introduction}

Nowadays, Most of oil reservoirs all over the worlds are showing a production declining by the time. As a results, most of them are in a highly needs to apply one of the enhanced oil recovery (EOR) methods. This may lead to produce additional quantity of crude oil from these reservoir rocks. Greater than about sixty percent of the crude oil may be kept in each reservoir after using these enhanced oil recovery. Therefore, there should be additional work to try to release such amount from these reservoirs [1] [2].

As it is well known these days, the stages of petroleum production from oil fields may be classified into phases. The first phase is primary recovery which can be done by natural flow or artificial lifting such as gas lifting and pumping. The second phase is secondary recovery which involves the injection of gas and/or water into the reservoir rocks. The final recovery factor may reach to more than $45 \%$. The main reasons for not getting higher recovery over that value are the capillarity, interfacial tension complication among the persistent fluids, reservoir heterogeneity, and mobility ratio (M). In general this is attributed to geological and physical factors. As a results, the remaining crude oil is the main objective for improved oil recovery (IOR) techniques. These techniques are normally applied for extracting more oil by changing oil or rock properties or both of them aiming to release much more crude oil from the reservoirs. From these methods, chemical flooding, thermal, and immiscible flowing operation.

The problems accounted in EOR applications have led the engineers scientists and oil producers to search for new methods of low cost, and low risk. Ultrasonic waves EOR technology is one of the techniques which may leads to reduce the remaining oil in the reservoir rock. Although this effect has been noticed, monitored and investigated previously but till now the main mechanism is not well known and defined, and hence it is not well understood.

In this paper, the impact of ultrasonic waves on the relative permeabilities of both water and oil as (a wetting and non-wetting) phases respectively is investigated through a series of experiments. Each experiment is accomplished in the presence and absence of ultrasonic 
waves. Then, using the data in each experiment, the wetting phase relative permeability curve is obtained. Finally, the relative permeabilities of the wetting and the non-wetting phases are plotted and compared in the presence and absence of ultrasonic radiation. Furthermore, it can be used to define and explore the recovery mechanisms for increasing oil recovery.

\section{Ultrasonic Waves and Vibration Effects}

The main mechanism after ultrasonic wave applications is highly complicated and so different philosophies have been established to expect the real cause of higher oil recovery factor. These waves deliver an energy to the crude oil particles to decrease capillary forces. This led to redistribution of the contained fluid in the cores and hence change the relative permeability curves. This effect could lead to mobilize the oil inside the sample and hence increase the ultimate recovery.

Several reasons could be responsible for improving the fluid flow in the reservoir rocks under ultrasound wave application. Capillary effect is also considered one of the main parameters that control the fluid flow in the rock. Hamida-Babadagli (2007) [3] identified and analyzed the effect of frequency, and radiation on capillary pressure. As a results, several experimental runs were conducted on several cores samples from Berea and Indiana limestone cores.

By inserting these cores in an ultrasonic chamber, and conducting the flowing process, the recovery factor was calculated. They found that ultrasonic waves improves capillarity of the rock samples. They claimed that this effect is depending on the fluid properties such as interfacial tensions and fluid density.

Hamidi et al. (2014) [4] investigated the effect of ultrasonic waves on the crude oil viscosity. Their work have been done using a frequency of 25 and $68 \mathrm{KHz}$. Their work was done under different temperature conditions. They indicated that the viscosity of the oil reduces after application of the ultrasonic waves. This is may attributed to the heat generated from application of such waves.

In 2014, Keshavarzia et al. [5] performed an experimental work using a glass bead pack porous medium. The runs were conducted with and without ultrasound waves of $22 \mathrm{KHz}$, and the relative permeability and the pertinent recovery were monitored. They used four different liquid in their works, these are kerosene, water, crude oil A and crude oil B. crude oil B is asphaltic crude oil. The final recovery factors were higher in all of these fluid after sonication except crude oil B. they attributed this to breakdown of asphaltene micelle in the oil which leads to viscosity increases and as a consequence permeability decrease. They used Hagoort method to calculate wetting phase relative permeability and Corey type relative permeability function in case of non-wetting phase relative permeability.

Hamida and Babadagli (2008) [6] investigated the effect of ultrasonic waves on fluid by pendant drop approach. Liquid water was flooded to a $0.1 \mathrm{~mm}$ Hastelloy C-276 capillary tube immersed in different oils. These liquids are oil and kerosene. The mean drop rate per minute was monitored for different wave intensity. They noticed that a peak drop rate at a specific intensity, which mainly based on viscosity and the interfacial tension among liquid water and the used oil. They concluded that the change in the interfacial tension may be the main results that leads to improve the oil recovery.

\section{Previous Lab and Field Trials}

Using ultrasonic waves to increase oil recovery began in 1950s [7]. The pioneer work was presented in 1965. Water flooding tests on core samples under ultrasonic energy with different frequencies $(1,3.1$, and $5.5 \mathrm{MHz})$ performed by Duhon and Campbell (1965) [8]. The injection was done close to the core center of the used sandstone core in which the ultrasonic probe was also presented. A delivery probe was located at the core end. Their runs displayed that the ultrasonic waves increased final recovery and displacement efficiency in these samples. Similar studies performed by Fairbanks and Chen (1971) [9], and Gadiev (1977) [10] applied ultrasound on saturated unconsolidated sand.

Current research works have described positive ultrasonic stimulation in some reservoir formations. Westermark et al. (2001) [11] in their work described and investigated the use of ultrasound to improve oil recovery in an oilfield in Osage County, Oklahoma.

The interest in elastic ultrasonic waves EOR technology returned to fifties of the last century. This interest is well demonstrated in the paper by Beresnev and Johnson (1994) in which they described the full spectrum of the work done in both the USSR and USA. They evaluated the efforts of over a hundred researchers searching the effects of vibrations from the ultra sound range of $5 \mathrm{MHz}$ to barely audible, low end of $1 \mathrm{~Hz}$ including traffic induced seismic stimulation. The effects of earthquakes on oil production were also reviewed, however the results of conventional and nuclear explosions were not $[11,12]$.

Westermark et al. (2001) [11] mentioned in their paper that the most of the published document originated from four Soviet institutions, each institute performed several paper on their laboratories. Therefore, the effect of these vibration ultrasound have been investigated and extended experimentally. The explanation of the effect of these waves have been characterized. While this period, several parameters have been raised to check and investigate such as wettability alteration, relative perm modification, water salinity and viscosity change. Nikolaevsky et al. (1996) [13] mentioned that the seismic waves produce seismic oscillations. The explained the theoretical and field investigations of the phenomena suggest that vibrations may influence substantially the water or oil relative permeability. That appears to be partially reconstituted at saturations that ordinarily would prohibit the flow of a particular phase.

Kouznetsova et al. (1998) [14] showed a great improvement the crude recovery and a significant decrease of the water oil ratio (WOR) by experimental work and pilot results. Soviet scientists developed seismic stimulation called vibro - seismic impact technology (VIST) that showed a good improvement in oil recovery. This 
technique is a surface stimulation approach. The waves make a great fasten for filtrating action, and of gravity segregation of the main fluid in the systems $[15,16]$.

Several Chinese investigators (1997) [ [17 -19] published their laboratory work on vibration stimulation, the tests have checked the effect of vibration on core plugs. Mingyuan (1999) [20] stated that the wettability of any sample may be modified to be water wet after using ultrasonic wave, i. e. after sonication. This may lead to improve and raise the ultimate recovery factor.

\section{Experimental Work}

\section{Cores and Equipment}

Several core samples were used and some of them were included and used through this study. These cores were cut in pairs; each two samples are from same homogeneous reservoir. Each core sample was cleaned and dried. These cores were then injected by brine water to immobile water saturation, then flooded by mineral oil until what we inject equal to what come out from the core. After that, the initial crude oil in place (OOIP) was monitored and calculated, i.e. the pore volume of the core sample. Length (L), diameter (D), pore volume (PV), bulk volume (BV), porosity $(\phi)$, and the absolute permeability $\left(\mathrm{K}_{\mathrm{a}}\right)$ for all of these core pluges are measured and presented in Table 1.

The experiments were conducted using mineral oil with viscosity $18 \mathrm{cp}$ and $29^{\circ} \mathrm{API}$ measured at ambient condition. The brine sample was used with $1.08 \mathrm{~g} / \mathrm{cc}$ density and $1.3 \mathrm{cp}$ viscosit at $25^{\circ} \mathrm{C}$. The apparatus used to generate and apply the ultrasonic wave called sonic viewer 170.

High Frequency Seismic Source: To apply vibration wave on the sample, a pulse generator is called "sonic viewer 170 (Model-5228)" was used to generate waves as shown in Figure 1. The apparatus is available in faculty of science, Ain Shams University, Egypt. An ultrasonic digital indicator consists of a pulse generator unit, transmitter and receiver transducers are used for sonic pulse velocity measurement. The transmitters and the receiver are positioned at the ends of the core sample and pulse wave velocity can be measured. The attachment of P-wave transducer and S-wave transducer to the core sample makes it easily feasible to measure P-wave along with Swave velocity. The integration of the external interfaces (RS-232C, GP-IB) as standard accessories, it versifies its function. Along with CRT screen display of data acquired by this instrument, its printer output, and its storage capacity.

Large part from this experimental work have been performed and published in SPE Kuwait Oil and Gas Show and Conference, 11-14 October, 2015 [1], and this paper is considered continuation for involving all commments of the conference and an update for the previous work.

\section{Experimental method}

The principle apparatus used in this study consists of flooding device and Ultrasonic producer (generator). The consequence of Ultrasonic was investigated on the core samples from an Egyptian oil reservoir. The flow diagram of the experimental procedures is depicted in Figure 2.

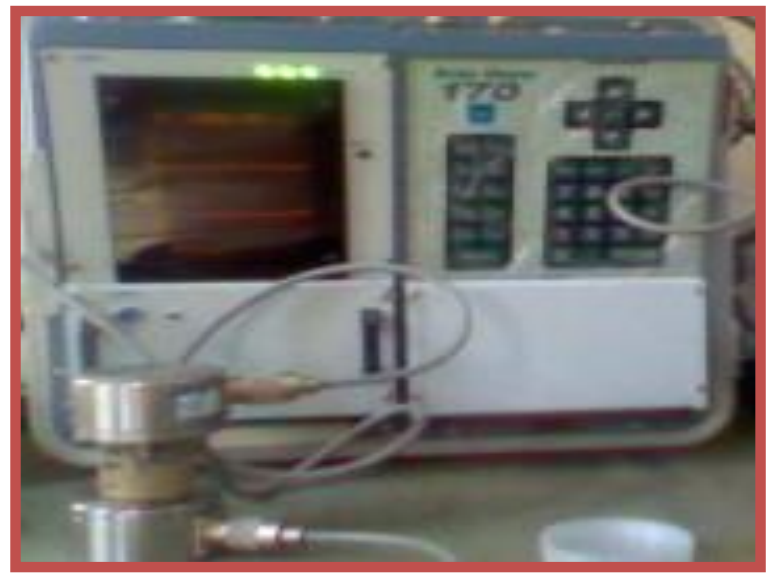

Figure 1 Sonic Viewer 170 (Model-5228)

Table 1 Core sample properties.

\begin{tabular}{c|c|c|c|c|c|c}
\hline $\begin{array}{c}\text { Core } \\
\text { No. }\end{array}$ & $\begin{array}{c}\mathrm{L}, \\
\mathbf{c m}\end{array}$ & $\begin{array}{c}\mathbf{D}, \\
\mathbf{c m}\end{array}$ & $\begin{array}{c}\mathbf{P V}, \\
\mathbf{c c}\end{array}$ & $\begin{array}{c}\mathbf{B V}, \\
\mathbf{c c}\end{array}$ & $\phi, \%$ & $\begin{array}{c}\mathrm{K}_{\mathrm{a}} \\
\mathbf{m d}\end{array}$ \\
\hline 1 & $\begin{array}{c}3.38 \\
3\end{array}$ & $\begin{array}{c}3.68 \\
2\end{array}$ & 8.63 & 36.04 & 23.95 & 460 \\
\hline 2 & $\begin{array}{c}3.38 \\
1\end{array}$ & 3.71 & 8.25 & 36.56 & 22.56 & $\begin{array}{c}305.1 \\
6\end{array}$ \\
\hline 3 & 4.89 & 3.79 & 7.47 & 55.19 & 13.54 & $\begin{array}{c}136.3 \\
1\end{array}$ \\
\hline 4 & 4.75 & 3.8 & 7.06 & 53.89 & 13.10 & 75.89 \\
\hline 5 & 4.83 & 3.93 & 13.43 & 58.61 & 22.91 & 67 \\
\hline
\end{tabular}

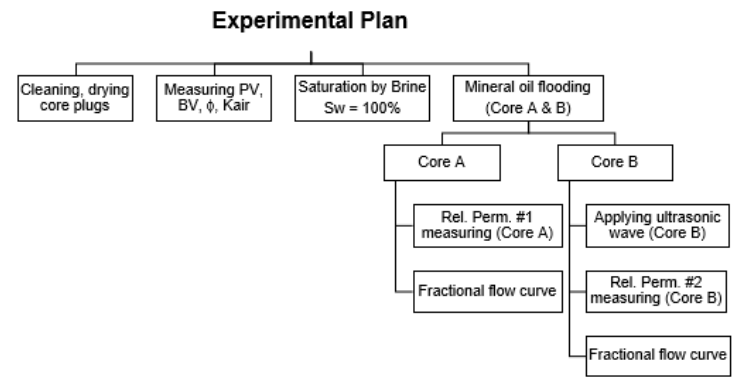

Figure 2 Experimental procedure plan

For getting the main objectives of the current work, the next laboratory steps were executed in a program to monitor the outcome of the ultrasonic on the relative permeability and the recovery factor of a reservoir as follows:

1. A pair of core samples (A and B) used in parallel, both of them is cut from the same level, i.e. having the same rock properties (same porosity, permeability, and wettability), and depicted in Table 1).

2. For each pair of core samples, rock and fluid properties were evaluated, first by evacuating by vacuum pump and soaked with water to determine pore volumes, bulk volume, and porosities. The cores absolute permeabilities were calculated and recorded.

3. These core plugs were injected with the mineral oil till reaching steady state. At this case, each core has initial water saturation $\left(S_{\text {wi }}\right)$ and certain oil saturation $\left(\mathrm{S}_{0}\right)$ which becomes like the reservoir. 
4. Using one of the cores of each pair (Core A), to monitor the relative permeability (Rel. Perm. \#I)

5. Applying water flooding in one of the other core sample (Core B) and record the output volume to calculate the recovery factor of water flooding $\left(R_{b}\right)$ recovery factor before ultrasonic application.

6. Applying ultrasonic waves by wave generator (at the residual oil saturation) of this core (Core B) and record the additional volume that can be obtained, calculate the recovery factor $\left(\mathrm{RF}_{\mathrm{a}}\right)$ after ultrasonic application.

7. Using core B, conduct, and plot the relative permeability curves after ultrasonic application (Rel. Per, \#II).

8. Comparing the two relative permeability curves obtained (Rel. Perm. \#1 and \#2).

\section{Determination of Relative Permeability}

The Benchtop Relative Permeameter system is designed to perform tests on core sample samples in order to determine liquid/liquid relative permeability. These tests were performed at ambient temperature with the unsteady-state technique. This system includes a fluid delivery pump, 2 piston accumulators, a core-holder, a back pressure regulator, a confining pressure system, and pressure measurement system. Operation of the system is controlled through a computer interface. The CYDAR software enables calculation of relative permeability. Preparation of the Test: loading the sample in the coreholder, initiating the confining pressure, initiating the back pressure, purging the lines, and pressure control. The relative permeability calculation and interpretation is performed using JBN method.

\section{Fractional Flow Equation}

The fractional flow equation predicts the relative amount of water that will flow at a given value of water saturation. The growth of the fractional flow equation is recognized by Leverett [21]. For two different liquid, oil and water, assuming there is a flow in a core sample or a reservoir, the water fraction at any point is named the fractional flow, $\left(f_{w}\right)$.

$$
f_{w}=\frac{q_{w}}{q_{w}+q_{o}}
$$

By neglecting the inclination angle and capillarity effect, the previous equation can me written as:

$$
f_{w}=\frac{1}{1+\frac{\mu_{w}}{\mu_{o}} \frac{k_{r o}}{k_{r w}}}
$$

This fw curve is used for designing the water flooding operation, such as frontal advance determination, average water saturation at the breakthrough time determination, and average water saturation at the front $\left(\mathrm{S}_{\mathrm{wf}}\right)$.

\section{Results and Discussion}

Ultrasonic Waves Applications
Sample \#1: Relative Permeability:

The relative permeability is measured two times, the first one is calculated before applying the ultrasonic on the core sample and the second one after applying this ultrasonic waves. The ultrasonic waves applied using ultrasonic source for about $90 \mathrm{~min}$. These measurements are calculated and interpreted by JBN methods and listed in Table 2. Then, the relative permeabilities are plotted versus the water saturation as depicted in Figure 3 and 4 for Sample \#1 before and after application of ultrasonic respectively.

Table 2 Relative perm. BEFORE and AFTER ultrasonic wave application for Sample \#1.

\begin{tabular}{ccc|ccc}
\hline \multicolumn{3}{c|}{ Rel. Perm. Before\#1 } & \multicolumn{3}{c}{ Rel. Perm. After\#1 } \\
\hline Sw & Kro_b & Krw_b & Sw & Kro_a & Krw_a \\
\hline 0.3526 & 1.0000 & 0.0000 & 0.3440 & 1.0000 & 0.0000 \\
\hline 0.3852 & 0.7520 & 0.0100 & 0.3852 & 0.7650 & 0.0100 \\
\hline 0.4026 & 0.6500 & 0.0150 & 0.4026 & 0.6700 & 0.0150 \\
\hline 0.4553 & 0.3900 & 0.0250 & 0.4553 & 0.4200 & 0.0250 \\
\hline 0.5026 & 0.1500 & 0.0300 & 0.5026 & 0.1700 & 0.0300 \\
\hline 0.5345 & 0.0750 & 0.0380 & 0.5300 & 0.1230 & 0.0370 \\
\hline 0.5523 & 0.0550 & 0.0430 & 0.5530 & 0.0900 & 0.0430 \\
\hline 0.5780 & 0.0350 & 0.0530 & 0.5790 & 0.0630 & 0.0520 \\
\hline 0.5972 & 0.0250 & 0.0600 & 0.5940 & 0.0510 & 0.0570 \\
\hline 0.6093 & 0.0200 & 0.0650 & 0.6100 & 0.0410 & 0.0630 \\
\hline 0.6196 & 0.0170 & 0.0700 & 0.6230 & 0.0350 & 0.0690 \\
\hline 0.6289 & 0.0140 & 0.0750 & 0.6340 & 0.0300 & 0.0740 \\
\hline 0.6346 & 0.0130 & 0.0780 & 0.6440 & 0.0260 & 0.0780 \\
\hline 0.6482 & 0.0100 & 0.0850 & 0.6600 & 0.0210 & 0.0860 \\
\hline 0.6521 & 0.0090 & 0.0870 & 0.6665 & 0.0190 & 0.0900 \\
\hline 0.6730 & 0.0000 & 0.0890 & 0.6960 & 0.0000 & 0.0930 \\
\hline
\end{tabular}

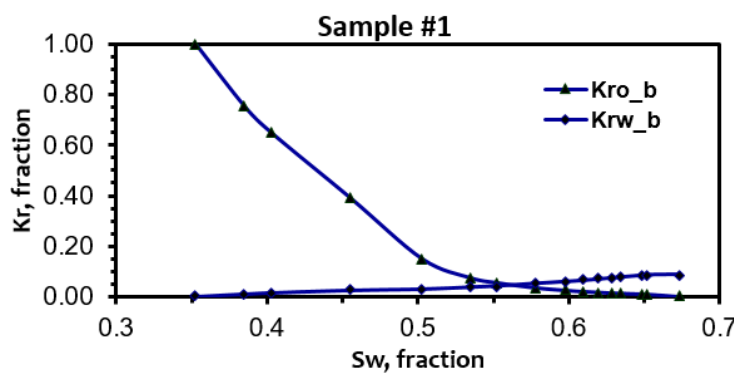

Figure 3 Relative permeability BEFORE ultrasonic application for Sample \#1.

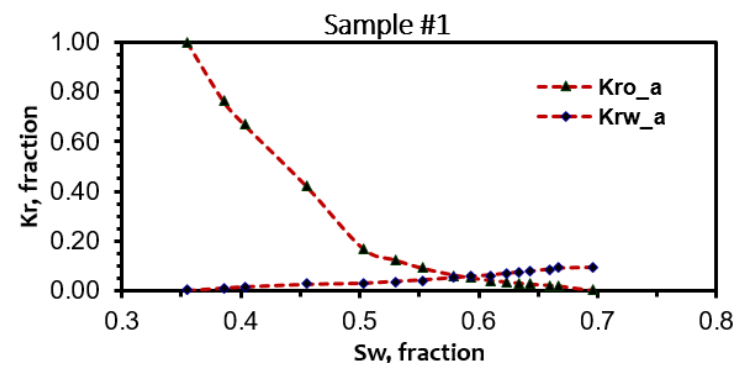

Figure 4 Relative permeability AFTER ultrasonic application for Sample \#1.

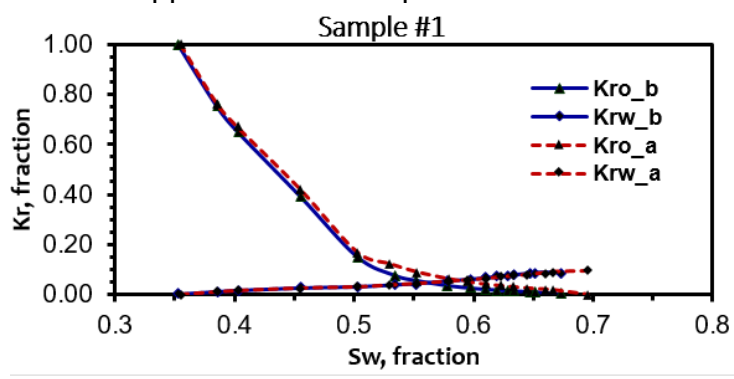

Figure 5 Relative permeability BEFORE and AFTER ultrasonic for Sample \#1. 
To investigate the effect of ultrasonic wave on the relative permeability curves, the two figure are overlapped shown in Figure 5 . It is noted that the ultrasonic waves modified the relative permeability. Moreover, the presence of ultrasonic waves caused an improvement of relative permeability to oil. For this sample, the improvement performed for the relative permeability to oil $\left(\mathrm{K}_{\mathrm{ro}}\right)$ in the saturation period from residual water saturation about $45 \%$ water saturation till residual oil saturation. But at less than $45 \%$ water saturation the relative permeability to oil in reversed. On the other hand, there is no improvement in the relative permeability to water. Since the curves of $\mathrm{K}_{\mathrm{rw}}$ are overlapped before and after the ultrasonic application.

Since this is the first run that shows the effect of ultrasonic is shown only for the relative permeability to water $\left(\mathrm{K}_{\mathrm{rw}}\right)$ only, it is needed to perform more experimental runs to investigate the real effect as it can be seen in the following sections.

\section{Fractional Flow Changes for Sample \#1}

The fractional flow ( $\mathrm{fw}$ ) equation predicts the relative amount of water that will flow at a given value of water saturation. It can be calculated using the previous equation. The effect of applying ultrasonic waves on fractional flow curve is depicted in Figure 6. the figures shows that the new curve after ultrasonic application becomes below that performed before the ultrasonic waves, this is mean that, by applying the rule for water saturation at the breakthrough, it will be greater than that calculated before, which means more oil will be produced and the recovery factor becomes higher that before. This is attributed to the vibration effect, shock waves formed after using high amplitude ultrasonic waves, heat generated that lowered the viscosity and the interfacial tension (IFT), and finally the caviataions.

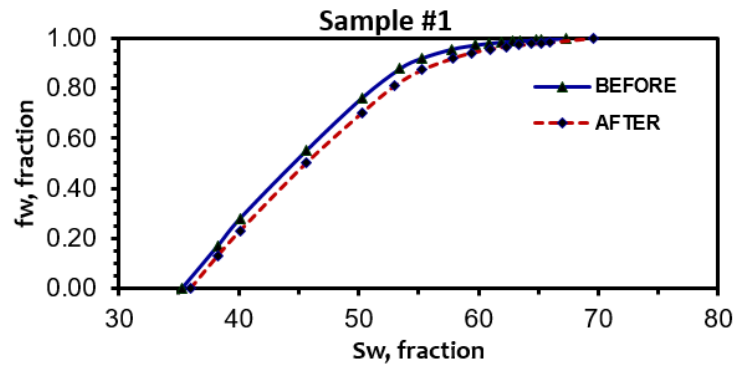

Figure 6 Fractional flow curve BEFORE and AFTER ultrasonic for Sample \#1.

\section{Sample \#2}

Relative Permeability: using Sample \#2 to check the effect of relative permeability deviations before and after applying ultrasonic waves. This core is having less permeability than that used in the first case. Its permeability $305 \mathrm{md}$, and its porosity is close to that of Sample \#1, about $23 \%$. The relatives permeabilities before and after are recoded and the data is listed in Table 3. These results are plotted and overlapped in the same plot as shown in Figure 7 for the relative permeability curve before and after applying the ultrasonic waves.
Table 3 Relative perm. BEFORE and AFTER ultrasonic wave application for Sample \#2.

\begin{tabular}{ccc|ccc}
\hline \multicolumn{3}{c|}{ Rel. Perm. Before \#2 } & \multicolumn{3}{c}{ Rel. Perm. After \#2 } \\
\hline Sw, frac & Kro_b & Krw_b & Sw, frac & Kro_a & Krw_a \\
\hline 0.3350 & 1.0000 & 0.0000 & 0.3440 & 1.0000 & 0.0000 \\
\hline $\mathbf{0 . 3 8 5 2}$ & 0.7520 & 0.0100 & 0.3852 & 0.7650 & 0.0100 \\
\hline $\mathbf{0 . 4 0 2 6}$ & 0.6500 & 0.0150 & 0.4026 & 0.6700 & 0.0150 \\
\hline $\mathbf{0 . 4 5 5 3}$ & 0.3900 & 0.0250 & 0.4553 & 0.4200 & 0.0250 \\
\hline $\mathbf{0 . 5 0 2 6}$ & 0.1500 & 0.0300 & 0.5026 & 0.2500 & 0.0300 \\
\hline $\mathbf{0 . 5 3 4 5}$ & 0.0700 & 0.0280 & 0.5300 & 0.1801 & 0.0370 \\
\hline $\mathbf{0 . 5 5 2 3}$ & 0.0400 & 0.0330 & 0.5530 & 0.0880 & 0.0430 \\
\hline $\mathbf{0 . 5 7 8 0}$ & 0.0310 & 0.0430 & 0.5790 & 0.0560 & 0.0520 \\
\hline $\mathbf{0 . 5 9 7 2}$ & 0.0250 & 0.0500 & 0.5940 & 0.0410 & 0.0570 \\
\hline $\mathbf{0 . 6 0 9 3}$ & 0.0190 & 0.0550 & 0.6100 & 0.0310 & 0.0630 \\
\hline $\mathbf{0 . 6 1 9 6}$ & 0.0150 & 0.0570 & 0.6230 & 0.0250 & 0.0690 \\
\hline $\mathbf{0 . 6 2 8 9}$ & 0.0130 & 0.0650 & 0.6340 & 0.0200 & 0.0740 \\
\hline $\mathbf{0 . 6 3 4 6}$ & 0.0120 & 0.0680 & 0.6440 & 0.0160 & 0.0780 \\
\hline $\mathbf{0 . 6 4 8 2}$ & 0.0110 & 0.0750 & 0.6600 & 0.0110 & 0.0860 \\
\hline $\mathbf{0 . 6 5 2 1}$ & 0.0080 & 0.0770 & 0.6665 & 0.0090 & 0.0900 \\
\hline $\mathbf{0 . 6 7 3 0}$ & 0.0000 & 0.0850 & 0.6960 & 0.0000 & 0.1100 \\
\hline
\end{tabular}

As shown in Figure 7, the relative permeability curves after ultrasonic application is moved to the right hand side. The cross-over point of the curve before the waves application is recoded as $56 \%$ water saturation, meanwhile the cross-over point moved to about 58\% water saturation. This imply the rock moves towards more water wetting condition. This result is coincident with that of Mingyuan et al. [20].

The relative permeability to oil $\left(\mathrm{K}_{\mathrm{ro}}\right)$ is lying above that done before the ultrasonic applications in all water saturation range from the beginning to the end. Which means a clear enhancement which could lead to increase the recovery factor from such reservoir.

\section{Fractional Flow Changes for Sample \#2}

Through the flooding of oil by water flood in this core, a rise in $\mathrm{fw}$ at any saturation in the sample will cause a proportional reduction in oil fractional flow $\left(f_{0}\right)$ and oil mobility. Consequently, the aim is to detect the correct flooding pattern that may reduce the water fractional flow. This can be reached by examining the effect of the injected water viscosity, inclination angle, and flooding rate on the water cut.

The enhancement obtained from relative permeability reflected here in the fractional flow curve as depicted in Figure 8. The fractional flow curve after stimulation by ultrasonic waves becomes lower that that performed before the ultrasonic wave application. Since the fractional flow of water, fw is distinct as the liquid water rate divided by the whole liquid rate either oil or water $\left(q_{t}\right)$, therefore, the water movements becomes less than before in the expense of oil movement that could increase the recovery factor after applying the ultrasonic waves.

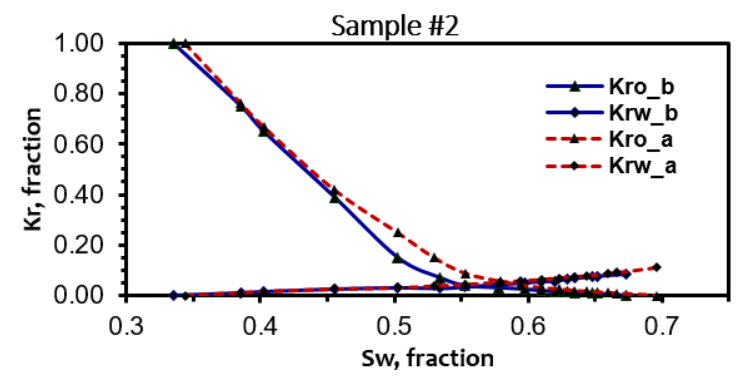

Figure 7 Relative permeability BEFORE ultrasonic application for Sample \#2. 


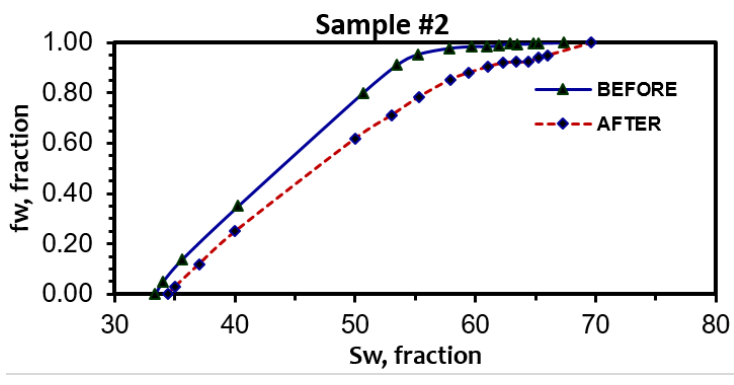

Figure 8 Fractional flow curve BEFORE and AFTER ultrasonic for Sample \#2.

\section{Sample \#3}

Relative Permeability: This time, Sample \#3 has used to check the effect of relative permeability change before and after applying ultrasonic waves. This core is having less permeability than that used in the first case. The relatives permeabilities before and after are recoded and the data is tabulated in Table 4. The results are plotted for the initial relative permeability and after applying the waves in the same graph, as shown in Figure 9.

By comparing the relative permeabilities curves as shown in Figure 9, the curve after ultrasonic application are shifted to the right, which means the cross-point moved to the right in which the water saturation increased. The cross-over point of the curve before the wave's application is recoded as $47 \%$ water saturation, meanwhile the cross-over point moved to about $50 \%$ water saturation. This implies that the rock moves towards more water wetting condition. This result coincides with that of Mingyuan et al. [20].

Table 4 Relative perm. BEFORE and AFTER ultrasonic wave application for Sample \#3.

\begin{tabular}{ccc|ccc}
\hline \multicolumn{3}{c|}{ Rel. Perm. Before \#3 } & \multicolumn{3}{c}{ Rel. Perm. Before \#3 } \\
\hline Sw & Kro_b & Krw_b & Sw & Kro_a & Krw_a \\
\hline 0.2235 & 1.0000 & 0.0000 & 0.2632 & 1.0000 & 0.0000 \\
\hline 0.3657 & 0.4040 & 0.0630 & 0.3812 & 0.3240 & 0.0390 \\
\hline 0.4295 & 0.2210 & 0.1120 & 0.4299 & 0.2070 & 0.0690 \\
\hline 0.4829 & 0.1390 & 0.1660 & 0.4743 & 0.1550 & 0.1110 \\
\hline 0.5320 & 0.0880 & 0.2120 & 0.5105 & 0.1290 & 0.1550 \\
\hline 0.5647 & 0.0620 & 0.2490 & 0.5675 & 0.0890 & 0.2400 \\
\hline 0.5892 & 0.0460 & 0.2870 & 0.6072 & 0.0570 & 0.3160 \\
\hline 0.6060 & 0.0370 & 0.3140 & 0.6232 & 0.0460 & 0.3480 \\
\hline 0.6318 & 0.0250 & 0.3470 & 0.6524 & 0.0280 & 0.4080 \\
\hline 0.6476 & 0.0190 & 0.3780 & 0.6840 & 0.0130 & 0.4620 \\
\hline 0.6599 & 0.0140 & 0.3920 & 0.7002 & 0.0060 & 0.4990 \\
\hline 0.6798 & 0.0070 & 0.4190 & 0.7216 & 0.0010 & 0.5570 \\
\hline 0.6979 & 0.0020 & 0.4410 & 0.7276 & 0.0010 & 0.5890 \\
\hline
\end{tabular}

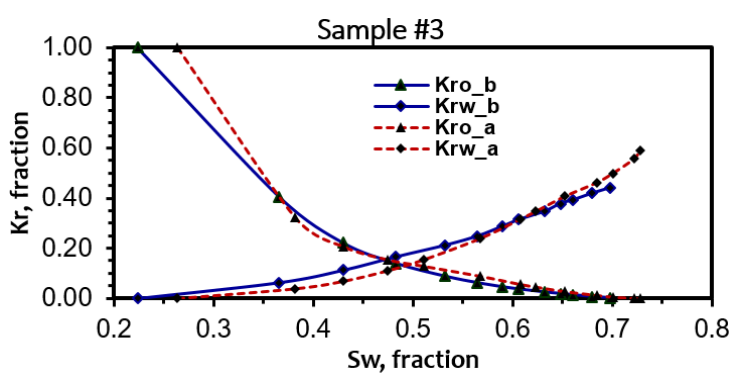

Figure 9 Relative permeability BEFORE and AFTER ultrasonic for Sample \#3.
It is noticed that the relative permeability to oil $\left(\mathrm{K}_{\mathrm{ro}}\right)$ is lying above that done before the ultrasonic application in almost all water saturation range (from $20 \%$ to $75 \%$ ). This means an improvement which could lead to increase the recovery factor. On the other side, it is shown that the ultrasonic waves lowered the relative permeability to water curve in approximately the entire saturation ranges of the experimental run. This means that the mobility of the water decreased after the wave application.

\section{Fractional Flow Changes for Sample \#3}

The improvement obtained from the relative permeability reflected here in the fractional flow curves as depicted in Figure 10. The fractional flow curve after stimulation by sonic waves becomes lower than that performed before the ultrasonic wave application.

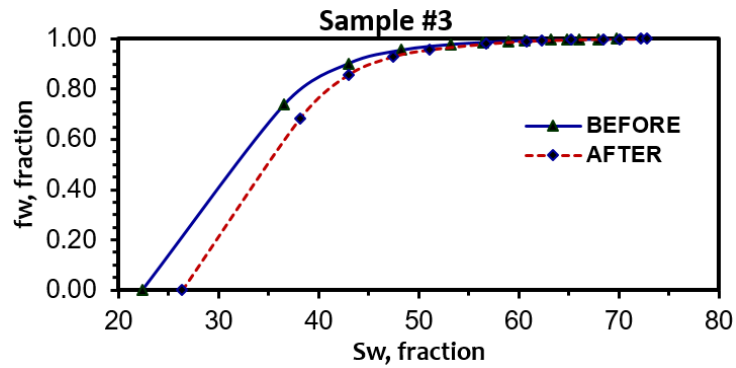

Figure 10 Fractional flow curves BEFORE and AFTER ultrasonic for Sample \#3.

\section{Sample \#4}

In case of Sample \#4, it has permeability lower than that of Sample \#3. Its permeability equals $75 \mathrm{md}$, and approximately have the same value of the previous sample. By measuring the relative permeabilities before and after ultrasonic application, the data are demonstrated in Table 5. Then plotting these curves on the same diagram as shown in Figure 11.

In this case there is no improvement in the relative permeability to oil to about $40 \%$ water saturation due to using ultrasonic waves. After that (i.e. from $\mathrm{S}_{\mathrm{w}}=$ $40 \%$ to the end), there is a small improvement in the relative permeability to oil. In other hand, the relative permeability to water decreased as a result of applying the ultrasonic waves from the beginning to the end, which lead to increase the recovery factor.

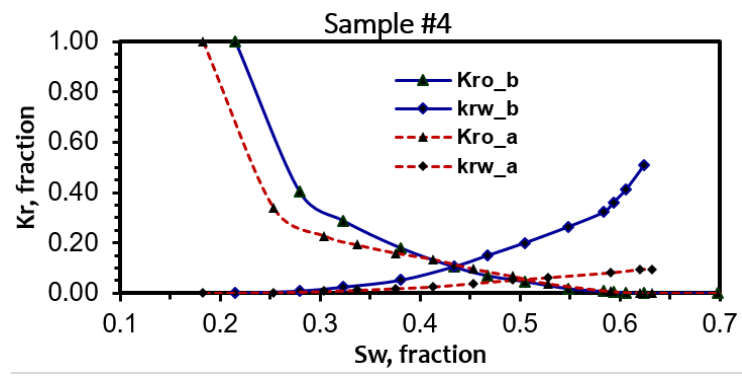

Figure 11 Relative permeability BEFORE and AFTER ultrasonic for Sample \#4. 
Table 5 Relative perm. BEFORE and AFTER ultrasonic wave application for Sample \#4.

\begin{tabular}{ccc|ccc}
\hline \multicolumn{3}{c|}{ Rel. Perm. Before \#4 } & \multicolumn{3}{c}{ Rel. Perm. Before \#4 } \\
\hline Sw, & Kro_b & krw_b & Sw & Kro_a & krw_a \\
\hline $\mathbf{0 . 2 1 5 2}$ & 1.0000 & 0.0000 & 0.1824 & 1.0000 & 0.0000 \\
\hline $\mathbf{0 . 2 7 9 3}$ & 0.4034 & 0.0092 & 0.2526 & 0.3413 & 0.0023 \\
\hline $\mathbf{0 . 3 2 2 7}$ & 0.2893 & 0.0245 & 0.3039 & 0.2286 & 0.0071 \\
\hline $\mathbf{0 . 3 8 0 2}$ & 0.1805 & 0.0518 & 0.3367 & 0.1928 & 0.0117 \\
\hline $\mathbf{0 . 4 3 3 7}$ & 0.1054 & 0.1054 & 0.3756 & 0.1600 & 0.0185 \\
\hline $\mathbf{0 . 4 6 7 1}$ & 0.0701 & 0.1496 & 0.4132 & 0.1350 & 0.0252 \\
\hline $\mathbf{0 . 5 0 5 0}$ & 0.0463 & 0.2004 & 0.4531 & 0.0970 & 0.0385 \\
\hline $\mathbf{0 . 5 4 8 1}$ & 0.0185 & 0.2636 & 0.4921 & 0.0680 & 0.0515 \\
\hline $\mathbf{0 . 5 8 3 7}$ & 0.0073 & 0.3221 & 0.5275 & 0.0387 & 0.0619 \\
\hline $\mathbf{0 . 5 9 3 1}$ & 0.0042 & 0.3597 & 0.5909 & 0.0078 & 0.0815 \\
\hline $\mathbf{0 . 6 0 5 8}$ & 0.0012 & 0.4110 & 0.6202 & 0.0021 & 0.0944 \\
\hline $\mathbf{0 . 6 2 4 3}$ & 0.0000 & 0.5094 & 0.6320 & 0.0009 & 0.0952 \\
\hline $\mathbf{0 . 6 9 7 9}$ & 0.0020 & & 0.7276 & 0.0010 & \\
\hline
\end{tabular}

Another conclusion that can get from the figure is that the cross-over point is moved from about $45 \%$ water saturation before applying the waves to about $51 \%$ water saturation. This means that the rock went to be more water wet than before, which coincide with Mingyaun et al. [20] findings. This is mean, increasing recovery factor as well.

\section{Fractional Flow Changes for Sample \#4}

The same result obtained as achieved in case of Sample \#3. The fractional flow curve goes down, below that obtained before ultrasonic wave application as depicted in Figure 12. Indeed, the flow of water is restricted and becomes below that got before ultrasonic wave application. By interpretation of those curves, the mean breakthrough water saturation after ultrasonic wave application is greater than that got before.

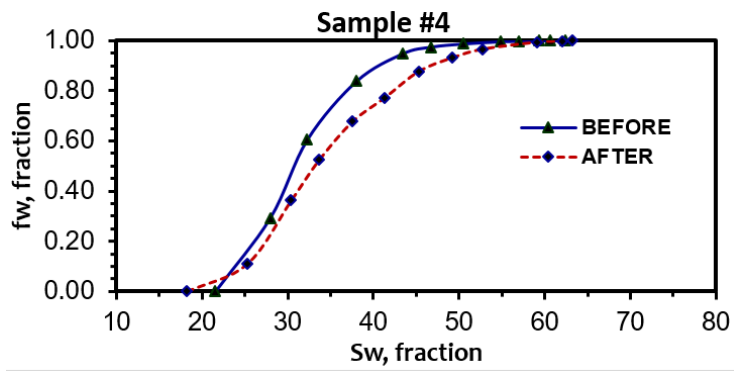

Figure 12 Fractional flow curves BEFORE and AFTER ultrasonic for Sample \#4.

\section{Sample \#5}

This sample has permeability value closed to that of Sample 4, about $67 \mathrm{md}$. The same technique applied for this sample as well, and the data is recorded and tabulated in Table 6. These data are plotted in Figure 13.

Table 6 Relative perm. BEFORE and AFTER ultrasonic wave application for Sample \#5.

\begin{tabular}{ccc|ccc}
\hline \multicolumn{2}{c|}{ Rel. Perm. Before \#5 } & \multicolumn{3}{c}{ Rel. Perm. Before \#5 } \\
\hline Sw & Kro & Krw & Sw & Kro & Krw \\
\hline $\mathbf{0 . 3 5 2 5}$ & 1.0000 & 0.0000 & 0.2662 & 1.0000 & 0.0000 \\
\hline $\mathbf{0 . 4 7 2 5}$ & 0.2514 & 0.0010 & 0.4252 & 0.2842 & 0.0078 \\
\hline $\mathbf{0 . 5 6 1 0}$ & 0.0729 & 0.0124 & 0.5118 & 0.1730 & 0.0322 \\
\hline $\mathbf{0 . 6 0 8 5}$ & 0.0277 & 0.0277 & 0.602 & 0.0646 & 0.0845 \\
\hline $\mathbf{0 . 6 3 0 8}$ & 0.0151 & 0.0332 & 0.6318 & 0.0440 & 0.1023 \\
\hline $\mathbf{0 . 6 4 5 9}$ & 0.0076 & 0.0371 & 0.6608 & 0.0236 & 0.1222 \\
\hline $\mathbf{0 . 6 6 7 3}$ & 0.0023 & 0.0488 & 0.7079 & 0.0081 & 0.1637 \\
\hline $\mathbf{0 . 6 8 7 2}$ & 0.0006 & 0.0565 & 0.7263 & 0.0041 & 0.1897 \\
\hline $\mathbf{0 . 6 9 9 1}$ & 0.0002 & 0.0643 & 0.7384 & 0.0018 & 0.2075 \\
\hline $\mathbf{0 . 7 0 2 3}$ & 0.0000 & 0.0662 & 0.7468 & 0.0007 & 0.2275 \\
\hline & & & 0.7554 & 0.0000 & 0.2555 \\
\hline
\end{tabular}

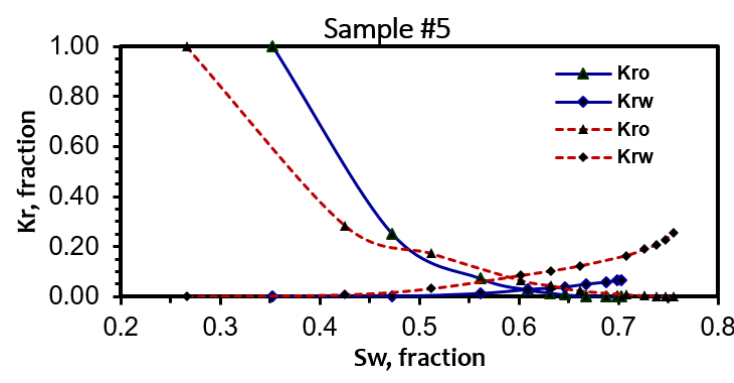

Figure 13 Relative permeability BEFORE and AFTER ultrasonic for Sample \#5.

The two relative permeability curves are displayed in Figure 13. It shows that the relative permeability to oil $\left(\mathrm{k}_{\mathrm{ro}}\right)$ decreased from the beginning to about $50 \%$ water saturation after using ultrasonic waves, then it improved till the end of the experiment. While, the relative permeability to water $\left(\mathrm{K}_{\mathrm{rw}}\right)$ after increased after using the ultrasonic waves from the beginning to the end of the run. Regarding to the wettability indication, the cross-over point moved from $61 \%$ to $59 \%$ water saturation. This is means that it goes towards oil wetting condition, which coincided with that of Mingyaun el al.[20] findings. This is of course reflected the recovery factor, it is expected to be lower than that got from previous runs.

\section{Fractional Flow Changes for Sample \#5}

In this sample, the discrepancy is very large at lower water saturation, i.e. at the beginning till about $60 \%$ water saturation. After that the two curves overlapped as presented in Figure 14, which means the improvement is less than that obtained from previous sample, this is confirm what we concluded in the interpretation of the relative permeability for this sample.

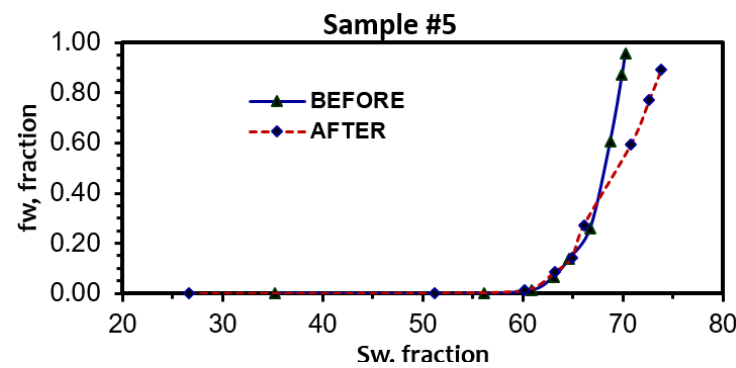

Figure 14 Fractional flow curves BEFORE and AFTER ultrasonic for Sample \#5.

After reviewing and analyzing all of the five samples, in terms of relative permeability change and fractional flow curve modification, the improvement achieved is attributed to many factors, these factor could be [22]:

1. Reduction of the interfacial tension with the frequency and time,

2. Reduction of the crude oil viscosity as a result of heat generated after ultrasonic application,

3. Oscillation and excitation of capillary confined oil drops due to forces created by mechanical vibration,

4. Emulsification,

5. Permeability increase attributed to pores restructure. 


\section{Conclusions}

This experimental work is considered one of the most important investigation that showed the various effects of ultrasonic wave applications to a real core sample from an Egyptian oil field, and the following are the main findings:

1. Ultrasonic wave improved the relative permeability curves and fractional flow curves for all permeable range under this study ( 67 to $460 \mathrm{md}$ ).

2. The improvement of ultimate recovery obtained in this study was under frequency of $500 \mathrm{KHz}$. This could suggest for a further work to widen the frequency ranges.

3. Depending on the intersection point of relative permeability curves, all of the core plugs become more water wet after ultrasonic waves.

4. Ultrasonic waves improved the oil recovery. This improvement may be due to wettability alteration, this change is noticeable in the permeability range 75 to $460 \mathrm{md}$.

\section{Acknowledgments}

The authors gratefully acknowledge the support of COREX lab engineers for their help and support. Highly appreciation to Prof. Abdel Moktader, Ain Shams University for his help.

\section{References}

1. Adel. M. Salem Ragab and M. Fouad Snosy, The Effect of Ultrasonic Waves of EOR on the Relative Permeability Curves. Society of Petroleum Engineers. doi:10.2118/175410MS, 2015.

2. M. Amro, M. A. Al Mobarky, and E. S. AlHomadhi, Improved Oil Recovery by Application of Sound Waves to Water Flooding, Society of Petroleum Engineers, SPE 105370, 2007.

3. T. Hamida, and T. Babadagli, Analysis of capillary interaction and oil recovery under ultrasonic waves, Transport in Porous Media journal, Vol. 70 issue 2 (November 2007) 231-255.

4. Hamidi, H., E. Mohammadian, E., Junin, 1 R., Rafati, R., Azdarpour, A., Junid, M., and Savory, R. M. : "The Effect of Ultrasonic Waves on Oil Viscosity," Petroleum Science and Technology, 32:2387-2395, 2014.

5. B. Keshavarzia, R. Karimi, I. Najafi, M. H. Ghazanfari, and C. Ghotbi, Investigating the Role of Ultrasonic Wave on Two-phase Relative Permeability in a Free Gravity Drainage Process, Scientia Iranica C, 21, issue 3, (2014) 763-771.

6. T. Hamida, and T. Babadagli, Effects of ultrasonic waves on the interfacial forces between oil and water, Ultrasonics Sonochemistry 15, Issue 4 (April 2008) 274278.

7. Khosrow Naderi, Core and Pore Scale Investigations on Immiscible Displacement and Enhanced Oil/Heavy-Oil Recovery under
Ultrasonic Waves, M.Sc. Thesis, University of Alberta, Edmonton, Alberta, 2008.

8. R. D. Duhon and J. M. Campbell, The Effect of Ultrasonic Energy on Flow through Porous Media, SPE 1316, $2^{\text {nd }}$ Annual Eastern Regional Meeting of SPE/AIME, Charleston, WV., 1965.

9. H. V. Fairbanks, and W. I. Chen, Ultrasonic Acceleration of Liquid Flow through Porous Media, Chemical Engineering Progress Symposium Series, 67: 105, 1971.

10. S. M. Gadiev, Use of Vibrations in Oil Production, (Ispol'zovaniye Vibratsii V Dobyche Nefti). Moscow, Nedra Press., 1977.

11. R. V. Westermark, J. F. Brett, and D. R. Maloney, Enhanced Oil Recovery with Downhole Vibration Stimulation, SPE 67303 presented at the SPE Production and Operations Symposium held in Oklahoma City, Oklahoma, (24-27 March 2001).

12. I. A. Beresnev et al., Elastic-wave Stimulation of Oil Production: A Review of Methods and Results, Geophysics, 59, No. 6, 1000, (June 1994).

13. V. N. Nikolaevskiy, et al., Residual Oil Reservoir Recovery with Seismic Vibrations, SPE Production \& Facilities 11 (2) (1996) 8994.

14. O. L. Kouznetsova et al., Improved Oil Recovery by Application of Vibro-energy to Waterflooded Sandstones, Journal of Petroleum Science and Engineering, 19 (1998) 191.

15. V. N. Belonenko et al., Vibro-seismic technology of hydrocarbon yield and analogies of the Australian Pacific region and CIS, Oil and Gas Australia, (July 1996).

16. V. N. Belonenko, Vibro Seismic Technology for Increasing Hydrocarbon Bed Recovery, New Technologies for the $21^{\text {st }}$ Century, Joint English/Russian Magazine, 14 (2000).

17. Y. Ling et al., Effects of Mechanical Vibration on the Capillary Pressure Curve and the Wettability of a Core, Xi An Shi You Xue Yau Xue Bao, 12 No. 5 (Sept. 1997) 23.

18. S. Changjin et al., Basic Research on Applying Physical Fields to Increasing Crude Oil Production, Shi You Xue Bao,18 No. 3 (July 1997) 63.

19. M. Jiangou et al., Increasing the Water Flood Recovery Efficiency of Cores by Mechanical Vibration, Xi An Shi You Xue Yau Xue Bao, 12 No. 4 (July 1997) 19.

20. L. Mingyuan et al., The Study of Oil Recovery by Water Flooding with Sound Vibration, Petroleum Science. 2 No. 2 (March 1999) 48.

21. Ahmed Tarek, Reservoir Engineering Handbook, $4^{\text {th }}$ Ed., Gulf Professional Publishing is an imprint of Elsevier, (2010).

22. T. Hamida, and T. Babadagli, Capillary Interaction of Different Oleic and Aqueous Phases between Matrix and Fracture Under Ultrasonic Waves, SPE-94105-MS, Society of Petroleum Engineers. (Jan. 2005). 